\title{
ESTUDIO EXPERIMENTAL SOBRE QUIMIOTERAPIA FOCALIZADA EN RIÑÓN MEDIANTE ARPÓN MAGNÉTICO Y ADMINISTRACIÓN INTRAVENOSA DE NANOPARTÍCULAS FERROCARBONOSAS.
}

\author{
José Gabriel Valdivia Uría",3, Manuel Ricardo Ibarra García',2, Rodrigo Fernández Pacheco', \\ Américo Viloria4, Teresa Higuera", Alicia Laborda4, Angel García 4 , José Antonio García de \\ Jalón", Martín Gutiérrez1,3, Maria Soledad Romero1,3, Rosa Cornudella3, Manuel Arruebo', \\ Clara Marquina², Jordi Arbiol5 y Jesús Santamaría?.
}

\author{
'Instituto Universitario de Investigación en Nanociencia de Aragón (INA). Universidad de Zaragoza. Zaragoza. \\ ${ }^{2}$ Instituto de Ciencia de Materiales de Aragón (ICMA). Universidad de Zaragoza-CSIC. Zaragoza. \\ ${ }^{3}$ Hospital Clínico Universitario Lozano Blesa. Zaragoza. \\ ${ }^{4}$ Departamento de Patología Animal. Facultad Veterinaria. Universidad de Zaragoza. Zaragoza. \\ 5TEM-MAT, Servicio Científico-Técnico (SCT). Universidad de Barcelona. Barcelona. España.
}

\begin{abstract}
Resumen.- OBJETIVO: El empleo de nanopartículas en el transporte de fármacos es actualmente uno de los temas de interés prioritario dentro del campo de la investigación biomédica. Nuestro objetivo es mostrar los resultados iniciales de un método inédito para focalizar en órganos sólidos nanopartículas ferro carbonosas quimioportadoras. Hemos obtenido y caracterizado diversos tipos de nanopartículas ferromagnéticas, y hemos estudiado su comportamiento in vitro e in vivo en animales de experimentación con dianas magnéticas intrarrenales implantadas laparoscópicamente.
\end{abstract}

MÉTODO: Obtenemos mediante arco de plasma nanopartículas ferro carbonosas capaces de absorber y desorber doxorrubicina y mostrar excelente comportamiento en estudios reológicos in vitro. Bajo anestesia general insertamos mediante control laparoscópico en

José Gabriel Valdivia Uría

$\frac{0}{U}$
$\frac{1}{d}$
$\frac{0}{0}$
$\frac{1}{0}$
$\frac{1}{0}$
0
$\frac{1}{0}$
$U$

Servicio de Urología

Hospital Clínico Universitario Lozano Blesa Avda. San Juan Bosco, 15

50009 Zaragoza. (España)

valdivia@unizar.es

Trabajo recibido: 18 de septiembre 2006. el riñón izquierdo de conejos de raza neozelandesa un microarpón magnético encapsulado en oro. En el mismo acto inyectamos por vía intravenosa diferentes dosis de distintos tipos de nanopartículas. Los diversos lotes de animales se sacrifican tras diferentes tiempos y se analizan histológicamente ambos riñones, bazo, hígado, pulmones y médula ósea.

RESULTADOS: Tras haber seleccionado las nanopartículas más adecuadas para nuestros fines, hemos constatado en los estudios post-mortem diferencias significativas en la distribución de las nanopartículas, con cúmulos de las mismas en la diana magnética y en el parénquima renal circundante. No obstante, el sistema retículoendotelial retiene gran parte de la carga inyectada.

CONCLUSIONES: Aunque nuestro sistema de focalización magnética resulta efectivo, deben ensayarse sistemas de blindaje temporal de las nanopartículas que les permita eludir la acción del sistema inmune.

Palabras clave: Carcinoma renal. "Drug carriers". "Drug delivery". Implante magnético. Nanopartículas magnéticas. Nanomedicina.

Summary.- OBJECTIVES: The use of nanoparticules for drug transport is one of the topics with priority interest within the field of biomedical research. Our objective is to show the initial results of an innovative method to focalize drug carrier ferro-carbon nanoparticules to solid organs. We obtained and characterized various types 
of ferrous magnetic nanoparticules and studied their behaviour in vitro and in vivo in laboratory animals with intrarenal magnetic targets laparoscopically implanted.

METHODS: Using a plasma arch we obtained ferro-carbon nanoparticules with the ability to absorb and deliver doxorubicin, showing an excellent behaviour in in vitro rheological studies. Under general anesthesia and control we inserted a gold covered magnetic microharpoon in the left kidney of New Zealand rabbits. At the same time we injected intravenously different doses of various types of nanoparticules. The animals were sacrified after pre-established times and pathologic studies of their kidneys, spleens, livers, lungs and bone marrow were carried out.

RESULTS: After selection of the most adequate nanoparticules for our purposes, we ascertained significant differences in the distribution of nanoparticules in post mortem studies, with accumulation in the magnetic target and surrounding renal parenchyma. Nevertheless, the reticuloendothelial system retains a great amount of the injected dose.

CONCLUSIONS: Although our magnetic focalization system is effective, nanoparticule temporary protection systems should be tested to allow us avoid the action of the immune system.

Keywords: Renal carcinoma. Drug carriers. Drug delivery. Magnetic implant. Magnetic nanoparticules. Nanomedicine.

\section{INTRODUCCIÓN}

El empleo de nanopartículas en el transporte y entrega de fármacos ("drug carriers" and "drug delivery") es actualmente uno de los temas de interés prioritario en el campo de la investigación biomédica. Con estos procedimientos se pretende obtener altas dosis de un fármaco en un punto determinado del organismo, reduciendo así sus efectos a nivel sistémico.

Las nanopartículas utilizadas para el transporte de fármacos son diminutas estructuras moleculares con tamaños inferiores a 200 nanómetros (*) que pueden ser "cargadas" con fármacos que deben "entregar" en el punto de destino por simple desorción pasiva o activa (mediante estímulo con fuentes de energía externa). La carga del fármaco puede situarse en el núcleo de la nanopartícula (es el caso de los liposomas), absorberse en su cubierta semiporosa (esto ocurre en las nanopartículas de carbón, sílice, etc.) o adherirse las ramas filamentosas de ciertas nanopartículas "funcionarizándolas" con ligandos químicos, físicos o inmunológicos.

(*) un nanómetro equivale a una millonésima de milimetro.
La vehiculización de fármacos mediante nanopartículas exige que éstas dispongan de una "información" adecuada sobre el punto al que deben acudir.

Este direccionamiento puede obtenerse de diversas formas: unas veces resulta espontáneo, por el simple estímulo mecánico que provoca la presencia de las nanopartículas, que como "cuerpos extraños" activan al sistema inmunocelular (es el caso de las nanopartículas superparamagnéticas de óxido de hierro, que tras ser inyectadas son rápidamente fagocitadas por los macrófagos y los linfocitos en las primeras estaciones linfáticas). Otra forma de direccionamiento es algo más sofisticada y requiere el recubrimiento de la superficie de las nanopartículas con receptores hormonales o anticuerpos específicos. Finalmente una posible tercera forma de direccionamiento de ciertas nanopartículas consiste en el aprovechamiento de la naturaleza magnética de su núcleo. En este sentido se han hecho ya ensayos clínicos experimentales utilizando grandes imanes externos para atraer nanopartículas ferromagnéticas hacia zonas superficiales para tratar tumores cutáneos (1-6). Este procedimiento requiere la inmovilización del paciente y sólo puede ser aplicable a campos muy superficiales (7-9). También se han diseñado posibles modelos teóricos empleando stents magnéticos (10-13).

Nosotros, basándonos en la buena tolerancia observada en la práctica clínica a diversos implantes y prótesis metálicas permanentes, y sirviéndonos de una larga experiencia en cirugía mínimamente invasiva (14). proponemos un método inédito, consistente en implantar por vía laparoscópica dianas magnéticas en el interior de órganos sólidos (en nuestro caso, el riñón) con el propósito de concentrar alrededor de las mismas, altas dosis de quimioterápicos vehiculizados mediante nanopartículas ferro carbonosas; todo ello en el supuesto de poder llegar a utilizar nuevos oncofármacos efectivos contra el hipernefroma.

\section{PRESENTACIÓN}

Nuestro equipo de investigación, integrado en el Instituto Universitario de Investigación en Nanociencias de Aragón, es de carácter multidisciplinar y cuenta con urólogos, físicos, químicos, veterinarios, oncólogos, hematólogos y patólogos. Desde hace dos años venimos trabajando en este proyecto de investigación que cuenta con la Ayuda Especial del Departamento de Ciencia, Tecnlogía y Universidad del Gobierno de Aragón para desarrollar proyectos de investigación y desarrollo tecnológico de carácter multidisciplinar. 
Como urólogos, sabemos que la quimioterapia sistémica, a dosis habituales, no resulta efectiva en pacientes con hipernefroma, a los que hasta el momento presente sólo podemos ofrecerles como tratamiento más eficaz la cirugía ablativa en sus diversas formas. No obstante, pensamos que el inconveniente de la toxicidad de la quimioterapia sistémica podría resolverse si se consiguiera una muy alta concentración de estos fármacos exclusivamente en área tumoral y escasa a nivel sistémico.

Nuestro equipo de físicos no sólo es capaz de producir nanopartículas ferro carbonosas, sino también que es capaz de caracterizarlas, seleccionando las de un tamaño apropiado como para ser inyectadas en el torrente sanguíneo, en una suspensión sonicada de gelafundina. Con el equipo de veterinarios de la Unidad de Técnicas de Mínima Invasión del Hospital Clínico Universitario Veterinario de Zaragoza decidimos que el animal idóneo para nuestros experimentos era el conejo de raza neozelandesa, entre otras cosas porque en él no tendríamos mayores problemas para la práctica de una laparoscopia.

El asesoramiento de los oncólogos y la posibilidad de estudiar el comportamiento en sangre de nuestras nanopartículas mediante estudios reológicos y de adhesividad plaquetaria, facilitó la realización de los estudios in vitro y ex vivo previos a la experimentación con animales vivos.

Finalmente la disponibilidad de un patólogo integrado en el equipo de investigación permitió un aprovechamiento máximo e integral de los estudios post mortem de los animales de experimentación.

\section{MATERIAL Y MÉTODOS}

\section{Obtención de nanopartículas}

La obtención de las nanopartículas de hierro recubiertas por carbono se realiza por dos procedimientos: mediante el horno de descarga de arco diseñado por Krätschmer-Huffman en 1990 (15) y mediante molienda mecánica por medio de un molino de bola de acero.

En el método de Krätschmer se utiliza una cámara cilíndrica con dos electrodos, un ánodo estático cargado con polvo de hierro y un cátodo movible de grafito, todo ello en atmósfera de helio. En adelante nos referiremos únicamente a este procedimiento, por su mejor rendimiento y la mayor homogeneidad de las muestras obtenidas.

Una vez purificadas las nanopartículas, se prepara con ellas un bioferrofluido, consistente en una suspensión al $1 \%$ de las partículas en gelafun- dina (gel comúnmente empleado como sustituto del plasma). Esta suspensión, en el momento de su preparación y antes de inyectarse es sometida a tratamiento con ultrasonidos durante varios minutos para una mejor dispersión de las partículas.

\section{Test in vitro}

La caracterización morfológica de las nanopartículas ferro carbonosas se realiza mediante experimentos de dispersión dinámica de luz (DLS), mediante microscopio electrónico de barrido (SEM), y microscopio electrónico de transmisión (TEM). Para apreciar el correcto recubrimiento carbonoso de las nanopartículas (y por tanto su biocompatibilidad), y la naturaleza del núcleo metálico de las partículas se recurre a al TEM de alta resolución (HRTEM) (Figura 1) y al TEM con filtro de energía (EFTEM), (16).

Las medidas de imanación de las nanopartículas y la caracterización magnética se realizan con un magnetómetro Squid (MPMM-5S), lo que nos permite seleccionar nanopartículas superparamagnéticas para nuestro estudio.

El cálculo de la superficie específica de nuestras nanopartículas capaz de absorber y desorber la doxorrubicina tiene un interés capital y se realiza con el método BET. Nuestras partículas presentan un área de absorción y desorción de $35 \mathrm{~m}^{2} / \mathrm{g}$ y de $14 \mathrm{~m}^{2} / \mathrm{g}$ respectivamente. Los valores no son excesivamente altos, sin embargo valores más bajos de superficie específica han sido utilizados para una adsorción eficaz y una desorción más rápida (17), por tanto consideramos que nuestras partículas son adecuadas para este propósito. La síntesis de nanopartículas encapsuladas en carbón activo para aumentar la capacidad de absorción del agente quimioterápico sería una forma efectiva de aumentar la superficie específica de estas partículas.

La cinética del proceso de adsorción de la doxorrubicina la medimos con el procedimiento descrito por Kuznetsov (18) incubando las nanopartículas ferro carbonosas en una solución de agua destilada con doxorrubicina, en tanto que el proceso de desorción lo estudiamos tratando de simular condiciones ex vivo, incubando las nanopartículas cargadas con doxorrubicina en plasma humano, a una temperatura constante de $36^{\circ} \mathrm{C}$ y bajo una ligera agitación.

Para analizar el comportamiento magnético de las nanopartículas en un medio coloidal, tratando de simular en cierto modo la resistencia a su translación que pudiera ofrecer el tejido intersticial de un ser vivo, realizamos suspensiones de las mismas en una película de agar (placas de Petri), a las que se les añade (como si se tratase de un antibioticograma) 

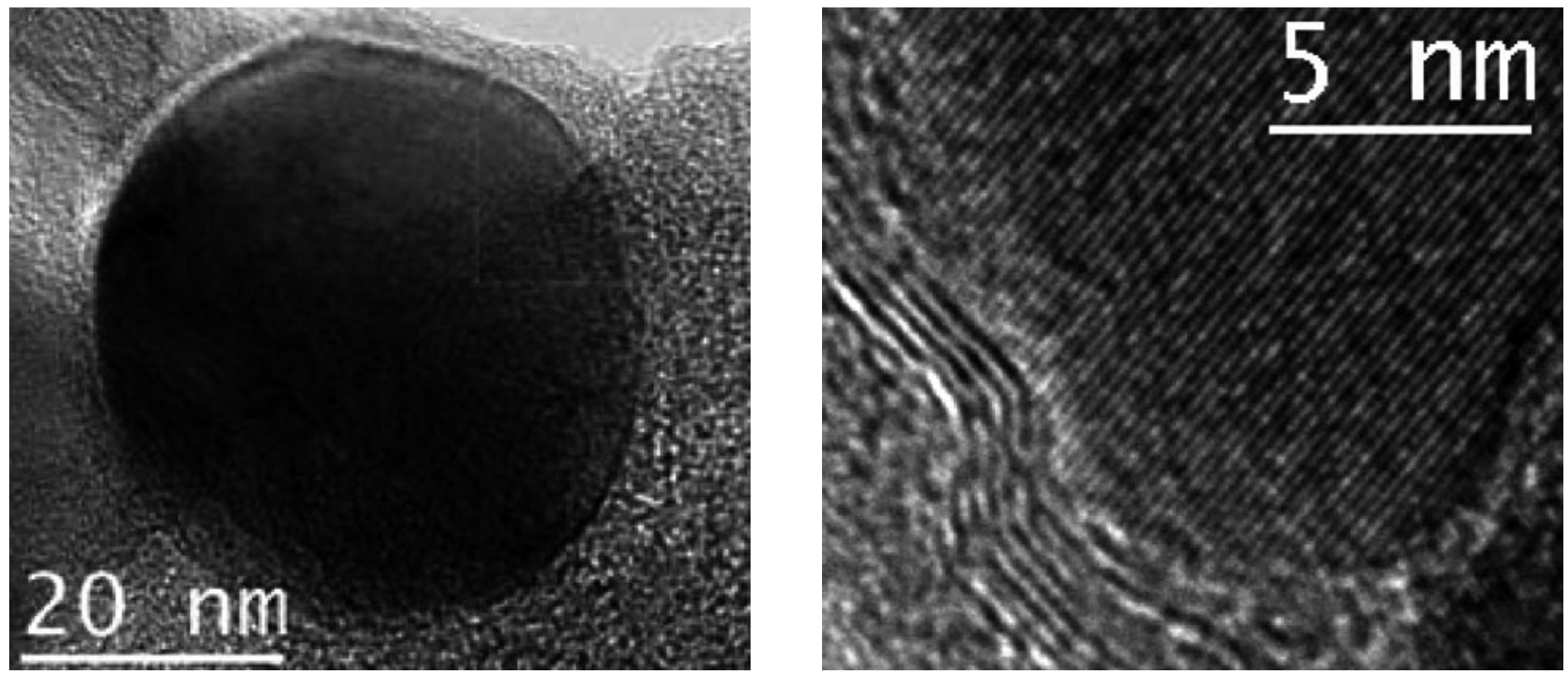

FIGURA 1. Imágenes obtenidas con microscopio electrónico de alta resolución (HTREM) de nanopartículas de hierro recubiertas por carbono.

una micro diana magnética en su centro. Seguidamente registramos mediante video digital los desplazamientos y la distribución de las nanopartículas alrededor de la diana magnética (Figura 2).

\section{Test ex vivo}

La norma ISO - 10993-4:2002 regula los tests de biocompatibilidad para los materiales que estarán en contacto con sangre circulante. Estos tests están diseñados para excluir la posibilidad de que se pudieran producir alteraciones celulares en los glóbulos rojos, blancos y en las plaquetas ocasionadas por el contacto con superficies extrañas, así como para detectar los posibles efectos adversos en el sistema de la coagulación.

Se utilizan para ello muestras de sangre humana proveniente de 5 voluntarios a las que se les añade distintas cantidades de ferrofluido $(1 \mathrm{ml}$ de sangre con $0.06 ; 0.12 ; 0.24$ y $0.5 \mathrm{ml}$ de ferrofluido). Con estas muestras se realizan estudios completos de coagulación y hemoreología (19).

Con el fin de analizar sin la interferencia del sistema retículo endotelial la distribución de las nanopartículas en ambos riñones procedemos a la inyección intraarterial de las mismas en un modelo experimental ex vivo. Para ello extraemos con técnica microquirúrgica, en el conejo anestesiado, un bloque anatómico que incluye a ambos riñones, la aorta y la cava. A uno de los riñones le insertamos un arpón magnético en su parénquima y al otro simplemente le infringimos un trauma similar en su corteza. Canalizadas la aorta y cava se establece mediante bomba de rodillos una perfusión continua de los riñones con solución de Ringer, a la que se añade $1 \mathrm{ml}$ de ferrofluido. Tras 10 minutos de perfusión, se interrumpe ésta y se remiten los riñones para estudio histopatológico.

\section{Test in vitro}

Utilizamos conejos de raza neozelandesa, 10 como control y otros 10 a los que se les inyecta por vía intravenosa $1 \mathrm{ml}$ de ferrofuido. Se realizan extracciones de sangre a los 10', 30' y 24 horas después de la inyección de las nanopartículas. Un conejo fue excluido del estudio porque el estudio post mortem mostró que padecía una neumonía.

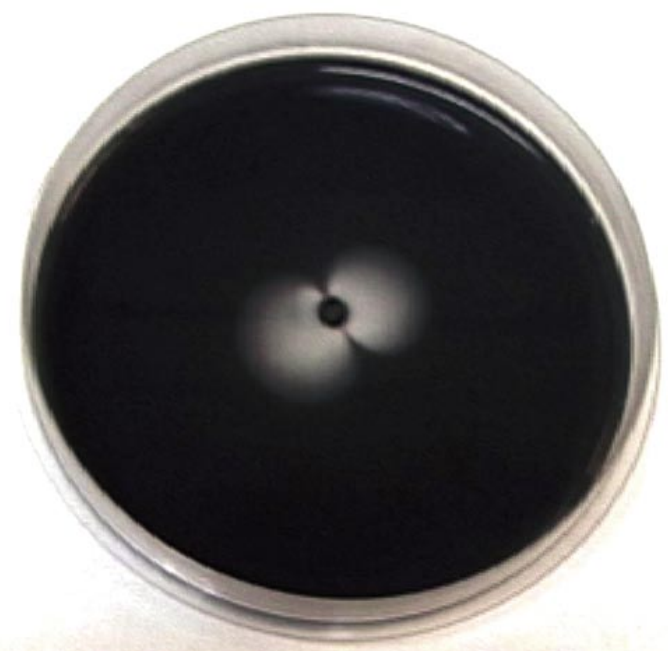

FIGURA 2. Placa de Petri con suspensión de nanopartículas de hierro tras haber colocado en su centro un pequeño imán. Obsérvese el halo de atracción magnética. 
En todos estos casos se realizan mediciones de la viscosimetría sanguínea con viscosímetro de Brookfield $\circledast$ y de viscosimetría de plasma con un viscosímetro capilar de Fresenius $\circledast$. Se determina también la agregabilidad de los eritrocitos con un agregómetro de Mirenne ${ }^{\circledR}$ y se realizan hemograma completo, recuento diferencial de leucocitos y test usuales de coagulación (20). Sin embargo, no hemos explorado una posible inhibición de la agregación plaquetaria ya que los tests que se emplean para ello no son de uso rutinario y sólo se utilizan con fines de investigación en patología humana. A pesar de ello, estamos tratando de modificar estas técnicas para hacerlas viables en sangre de conejo y poder así incluir este factor en nuestra investigación.

\section{Modelo quirúrgico experimental in - vivo}

Con el fin de poder analizar la tolerancia y el comportamiento de las nanopartículas ferro carbonosas en un ser vivo, así como poder objetivar la distribución de las mismas en diversos órganos, tejidos y preferentemente en un órgano par (considerado como órgano diana) que permita un análisis comparativo, diseñamos con la aprobación del Comité Ético para la Experimentación Animal, de la Universidad de Zaragoza (código PI 19/04), un modelo quirúrgico que consideramos viable, reproducible y mínimamente invasivo.

Para reducir el número de animales de experimentación se realizaron previamente los ya citados estudios in vitro y ex vivo. No obstante, al no existir ningún otro método alternativo capaz de mostrar la tolerancia, comportamiento, distribución y posibles efectos adversos de las nanopartículas, la necesidad de llevar adelante el estudio in vivo resulta incuestionable.

Los animales, tras un ayuno de 12 horas, son premedicados con una inyección intramuscular de Medetomidina (Domtor $\circledast$ ) $10 \mathrm{mg} / \mathrm{kg}+$ Ketamina (Imalgene $® 500$ ) $20 \mathrm{mg} / \mathrm{kg}$. Solo en caso necesario se administra complementariamente Atropina 0,05 $\mathrm{mg}$, también por vía intramuscular.

Se cateteriza la vena marginal de la oreja con catéter Vasocan Braunüle de 22 gauge, con el fin de perfundir suero fisiológico y dosis complementarias de anestésicos si fuera preciso y el ferrofluido (nanopartículas).

Transcurridos 5 minutos, se les intuba con traqueotubo de silicona con neumotaponamiento, $\mathrm{n}=2,5$ a 3,5 (provisto de fiador), guiados por un laparoscopio rígido y en posición de decúbito esternal. Si con la dosis intramuscular de anestésico no se detiene el reflejo laríngeo a la hora de intubar, se administran intravenosamente 0,5-2 $\mathrm{ml}$ de Propofol. Se conecta el traqueotubo a un circuito anestésico tipo T de Ayre y se suministra Isofluorano $(0,8-1 \%)$, vehiculizado en oxígeno $0,400-\mathrm{l} / \mathrm{m}$, con el fin de mantener la anestesia.

Durante el periodo postoperatorio se administra antibioterapia con Enrofloxacina (Alsirß) 5 $\mathrm{mg} / \mathrm{kg}$ subcutáneo y analgesia con Meloxican 0.3 $\mathrm{mg} / \mathrm{kg}+$ Buprenorfina $0,05 \mathrm{mg}$ por vía subcutánea. Estos fármacos se administran en una sola dosis, salvo en los casos en los que se practica laparotomía, en los que se administran durante 4 días).

Tras ser rasurados y anestesiados los animales son colocados posición supina. Se aseptiza la piel del abdomen, se aísla el campo quirúrgico y se realizan dos pequeñas incisiones cutáneas, de $5 \mathrm{~mm}$, una a nivel del ombligo y otra en fosa iliaca izquierda. Por el ojal cutáneo del ombligo se inserta una aguja de Veress y se distiende el abdomen con $\mathrm{CO}_{2}$ hasta alcanzar una presión de $15 \mathrm{~mm}$ de $\mathrm{Hg}$. Se introduce entonces por cada ojal cutáneo un trócar laparoscópico corto, de $5 \mathrm{~mm}$ de diámetro. La óptica laparoscópica de cero grados se introduce por el trócar umbilical para realizar una primera inspección laparoscópica del abdomen. Se lateraliza al animal hacia un decúbito lateral derecho, para descubrir el borde externo y cara anterior del riñón, recubierto parcialmente por la grasa de Gerota y la reflexión cólica. Por el trócar de la fosa iliaca se introduce un punzón de material plástico, de 8 French, provisto de camisa semitransparente. Se punciona con el mismo el polo inferior del riñón y se profundiza en el mismo siguiendo una dirección craneal y paralela al borde externo del parénquima (Figura 3). Tras haberse alcanzado una profundidad de $15 \mathrm{~mm}$ se retira el fiador del punzón y se pasa por el interior de su camisa

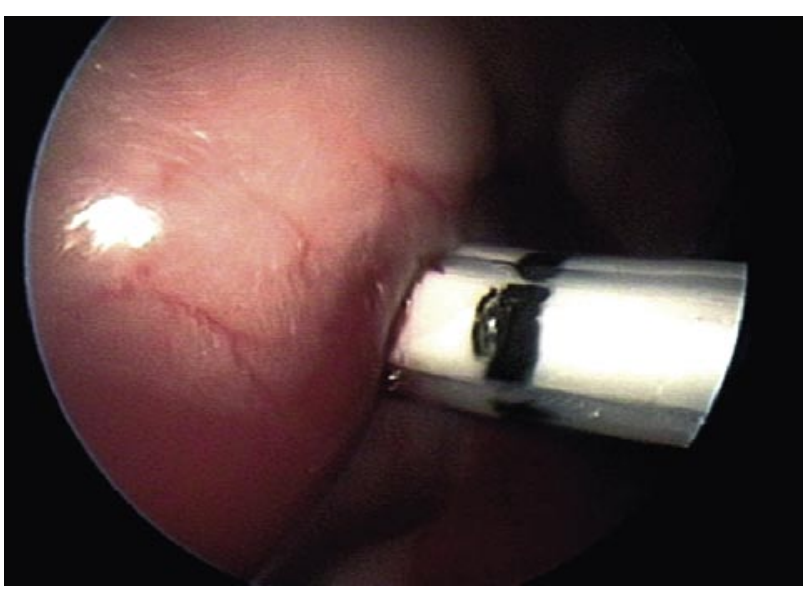

FIGURA 3. Implante de diana magnética en riñón izquierdo de un conejo bajo control laparoscópico. 
el imán dorado, el cual es empujado hacia el interior del parénquima renal con el propio fiador del punzón de plástico. Tras ello se retira el punzón, se lateraliza al conejo hacia un decúbito lateral izquierdo. Bajo control óptico se avanza con el trócar laparoscópico de fosa iliaca izquierda hacia el riñón derecho, salvando la posible interferencia del paquete intestinal. Se introduce entonces el punzón y se repite la misma maniobra de punción en el riñón derecho, pero sin dejar implantado ningún material en el mismo.

Comprobada la ausencia de hemorragia, se vacía el $\mathrm{CO}_{2}$ y se extraen los trócares cerrándose los orificios parietales con grapas metálicas.

Se procede entonces a administrar intravenosamente el ferrofluido a través de la vena marginal de la oreja del animal (Figura 4).

\section{Sacrificio, exámenes laparotómico y necrópsico y ex- tracción de órganos}

A todos los animales, incluidos los de los grupos testigo, se les somete a segunda anestesia general, esta vez con carácter terminal, a las 24 horas, - a los 7 ○ 30 días de la primera. Tras extraerles sangre para análisis se practica una inspección laparotómica in vivo con el fin de examinar los órganos diana, así como el conjunto de órganos vitales y se les provoca la eutanasia por sobredosis de anestésico (2 gramos de Tiopental Sódico.). Se extraen primero el riñón izquierdo (evitando en su proximidad el uso de instrumentos metálicos), luego el derecho, el hígado, bazo, pulmón y médula ósea. Se remiten, por separado y debidamente señalizados para su estudio anatomopatológico.

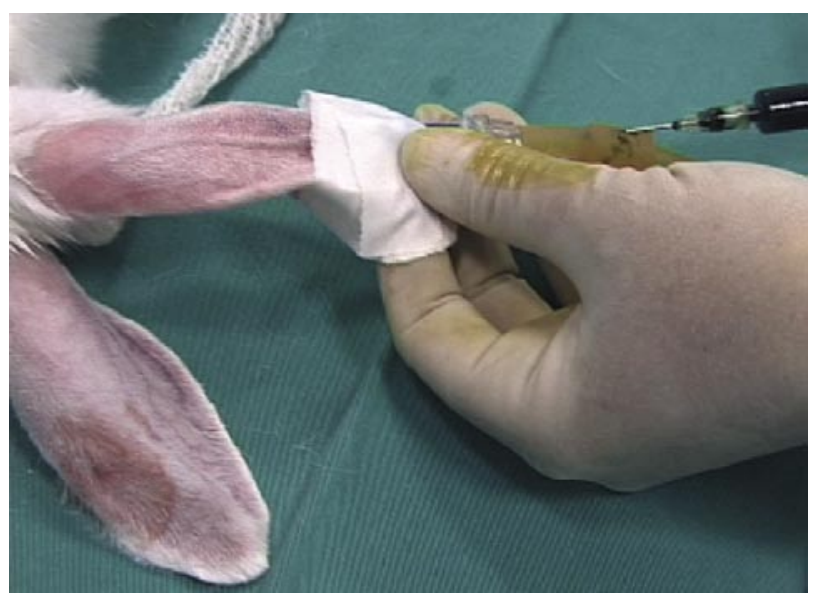

FIGURA 4. Inyección de $2 \mathrm{ml}$ de ferrofluido (nanopartículas de hierro) en la vena marginal de la oreja de un conejo.

\section{Estudio anatomopatológico}

Los órganos y muestras se fijan en formaldehído al $10 \%$ y se procesan rutinariamente, poniendo especial cuidado en no desplazar de su ubicación al imán implantado en el riñón izquierdo, el cual, al igual que el contralateral son abiertos coronalmente en dos mitades. Tras ello se obtienen macrofotografías de ambos (Figura 5).

La valoración histopatológica se basa en secciones de estas muestra teñidas con Hematoxilina y Eosina. Se obtienen microfotografías a 200 y 400 aumentos (Figuras 6, 7 y 8 ).

\section{RESULTADOS}

Utilizando el método de Krätschmer hemos conseguido resultados más uniformes en cuando a tamaño y morfología de las nanopartículas de hierro. Su caracterización morfométrica mediante dispersión dinámica de luz demuestra que su tamaño hidrodinámico en suspensión no supera los 200 nanómetros tras haber sido recubiertas por carbono.

Las nanopartículas obtenidas mediante molino atómico tienen una forma astillada e irregular, lo que las hace más propensas a ocasionar trastornos reológicos.

La suspensión de las nanopartículas en gelafundina ha demostrado ser un procedimiento idóneo para preparar un ferrofluido estable y apto para ser inyectado intravenosamente sin que se produzca precipitación de su soluto.

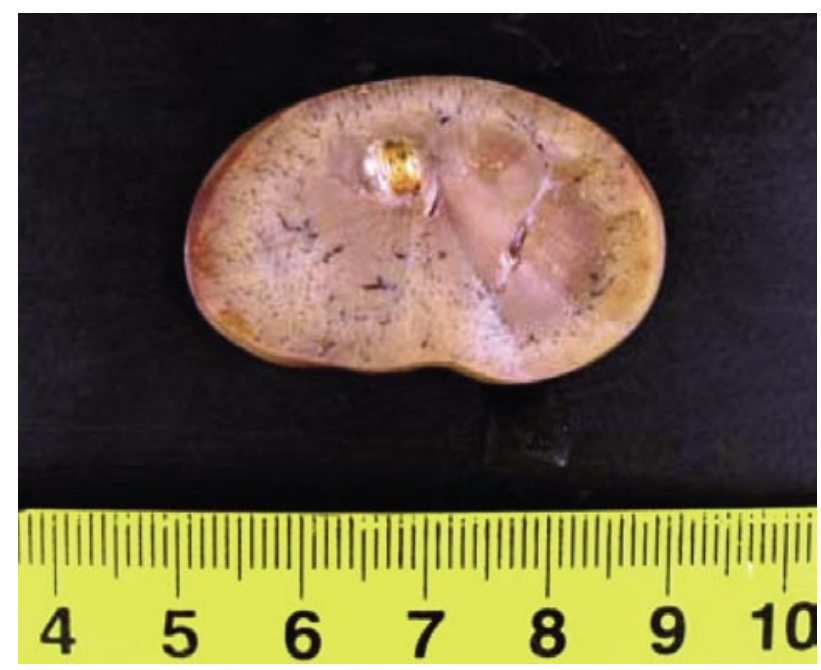

FIGURA 5. Corte coronal de riñón izquierdo de conejo, siete días después de habérsele implantado la diana magnética (excelente tolerancia local). 


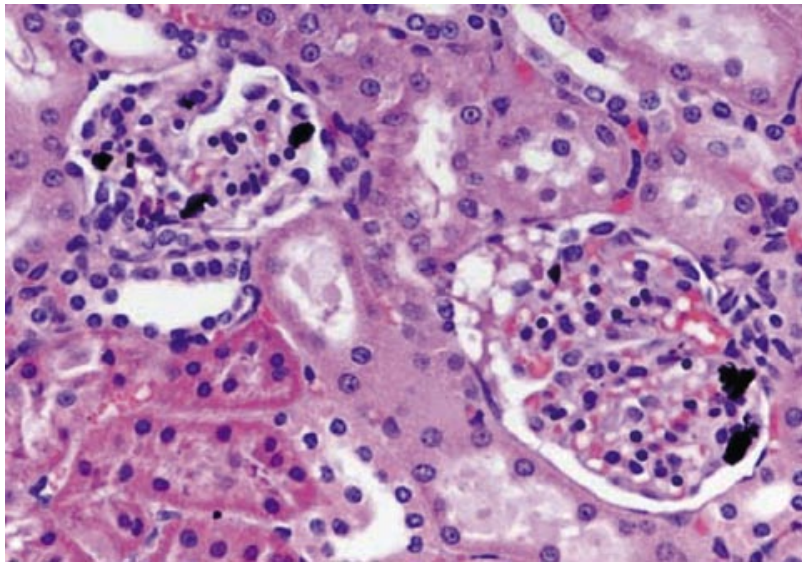

FIGURA 6. Imagen histopatológica de riñón izquierdo con acúmulos de nanopartículas de hierro. Hematoxilina Eosina $400 x$.

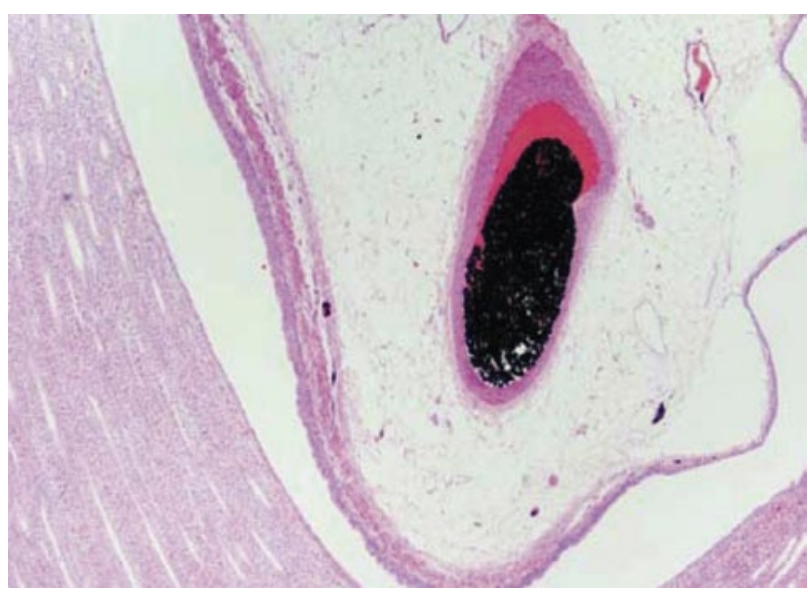

FIGURA 7. Imagen histopatológica de riñón izquierdo a 40 aumentos. Arteriola próxima a la diana magnética con émbolo de nanopartículas de hierro.

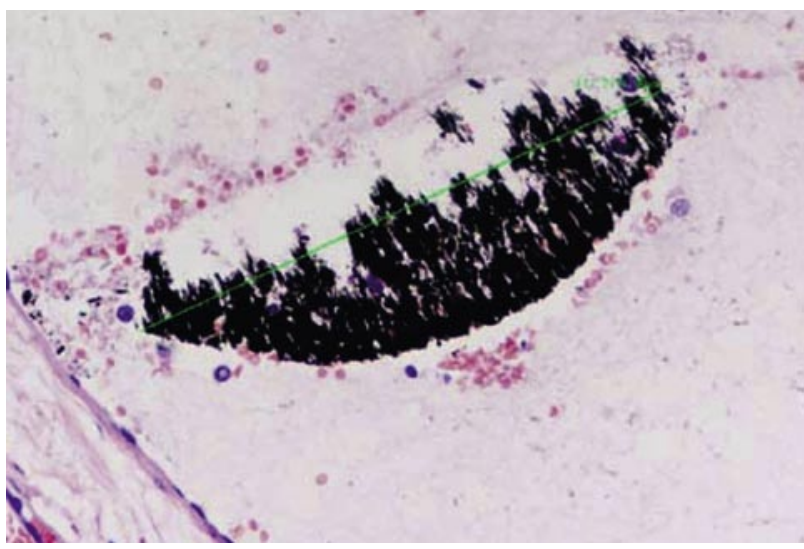

FIGURA 8. Imagen histopatológica de riñón izquierdo a 400 aumentos. Vaso próximo al imán, ocupado por nanopartículas que muestran polarización magnética.
La caracterización con HRTEM y EFTEM de las nanopartículas de hierro recubiertas de carbono obtenidas en nuestro laboratorio permite comprobar que el recubrimiento carbonoso de las mismas en completo, lo cual garantiza no solo su biocompatibilidad, sino que asegura la efectividad en el proceso físico de la adsorción de la doxorrubicina. Además, las capas de carbón aíslan a unas partículas de otras, evitando los posibles problemas de agregación causados por la interacción entre partículas magnéticas. Hemos comprobado asimismo que las capas de carbón evitan la oxidación y degradación que inexorablemente se produce en nanopartículas desnudas de hierro inmersas en medios acuosos.

La medición de la superficie específica de absorción de nuestras nanopartículas, realizada con el método $B E T$, muestra que éstas son perfectamente útiles para el transporte de fármacos, pues ofrecen una superficie de absorción de $35 \mathrm{~m} 2 / \mathrm{g}$ y de desorción de $14 \mathrm{~m}^{2}$. En un futuro, con el propósito de incrementar aún más la superficie de esta área específica existe la posibilidad de sintetizar partículas recubiertas de carbón activo, con lo que pensamos que podremos incrementar la carga farmacológica transportada.

En ensayos in vitro hemos podido constatar que el comportamiento magnético de nuestras nanopartículas en un medio coloidal es excelente, siempre que éstas se encuentren dentro del radio de atracción magnética del micro imán $(4 \mathrm{~mm}$ de diámetro $\times 2 \mathrm{~mm}$ de altura) y que es de alrededor de $10 \mathrm{~mm}$.

Nuestras partículas ferro carbonosas han mostrado ser perfectamente biocompatibles en los diferentes tests hematológicos y reológicos realizados tanto con sangre humana como en la de los animales de experimentación (conejos).

Para estudiar la distribución de las nanopartículas en ambos riñones del conejo, eludiendo artificialmente las barrera que supone el sistema retículo endotelial, diseñamos un modelo de bloque anatómico ex vivo sometido a una perfusión intravascular con suero Ringer al que se habían añadido nanopartículas ferro carbonosas. Este estudio nos permite apreciar una muy clara distribución preferente de nanopartículas hacia el riñón que porta el arpón magnético, resultados objetivados mediante estudio histopatológico de ambos órganos.

Consideramos de gran interés, puesto que no se había hecho antes, el estudio de la distribución, comportamiento y tolerancia de las nanopartículas en un ser vivo tras serle implantada una diana magnética en el interior de un órgano sólido profundo, como es el caso del riñón. 
En este sentido, el modelo quirúrgico diseñado al efecto, es mínimamente invasivo y se muestra altamente eficaz en este tipo de animales. Hemos implantado con éxito la diana magnética en 40 conejos. En 36 casos el implante se llevó a cabo en el riñón izquierdo y en 4 en el derecho debido a problemas técnicos que impidieron su correcta implantación en ese lado y se hizo preciso hacerlo en el contralateral, especificándose este incidente en el protocolo quirúrgico. En todos los casos se realizó el mismo trauma quirúrgico (punción con punzón de $12 \mathrm{Fr}$ ) en el riñón opuesto con el fin de que ambos riñones tuvieran una hemorragia intraparenquimatosa y no produjera una interpretación errónea de los hallazgos histopatológicos.

En 38 casos la técnica se llevó a cabo mediante el procedimiento laparoscópico previamente descrito. En otros dos animales se llevó a cabo mediante laparotomía media supra e infraumbilical.

En 37 animales el ferrofluido se administró por vía intravenosa a través de la vena marginal de la oreja. En 3 animales el ferrofluido se administró en un vaso más central, a través de una mini incisión cutánea en el cuello (en uno en la vena subclavia y en dos en la arteria carótida primitiva). A 36 animales se les inyectaron 2 mililitros de ferrofluido al $1 \%$. En otros 3 se administraron dosis de $10 \mathrm{ml}$ al $1 \%$ de ferrofluidos comerciales y en otro una dosis de $10 \mathrm{ml}$ al $2 \%$ de nuestro bioferrofluido.

En 3 casos se produjo la muerte intraoperatoria del animal tras haber implantado ya la diana magnética. La muerte se produjo en 2 casos por fallo anestésico (un caso por acodamiento del traqueotubo y otro por deshinchado accidental del balón del mismo) En otro caso el fallecimiento se produjo tras la inyección de una dosis excesivamente alta de ferrofluido (10 ml).

En otros 2 casos se produjo la muerte de los animales en el postoperatorio inmediato. En uno de ellos la autopsia inmediata demostró la presencia de una hemorragia procedente del riñón al que se había colocado el implante. Esto no fue la causa de la muerte del otro animal, por lo que cabe atribuirla a un posible espasmo laríngeo que pasó inadvertido.

Los 35 animales supervivientes fueron sacrificados tras ser sometidos a una nueva anestesia general terminal. A 20 conejos se les sacrificó a las 24 horas del implante en tanto que a 13 se los sacrificó a la semana de la intervención. A 2 conejos (que fueron intervenidos mediante laparotomía) hubo que sacrificarlos al $3^{\circ}$ y $5^{\circ}$ día por haberse detectado signos de una posible evisceración incipiente.
En ningún caso se desaprovecharon los órganos, y en todos pudo realizarse el oportuno estudio histopatológico.

\section{DISCUSIÓN}

Está plenamente aceptada la muy escasa efectividad de la quimioterapia en los tumores renales. Los fármacos más comúnmente empleados como la vimblastina y la floxuridina han mostrado tasas de respuesta de tan solo un 7 y $16 \%$ respectivamente. En un estudio en fase II realizado sobre 3.500 pacientes se observó una tasa de respuesta objetiva del $5,6 \%$, tras haber utilizado 72 diferentes fármacos antitumorales (21). En los últimos años tampoco se han llevado a cabo avances significativos en este terreno (22).

Por una parte está la marcada toxicidad de estos fármacos cuando se pretende utilizarlos a dosis elevadas, incluso administrándolos en esquemas de infusión continua ajustados al ritmo circadiano $(23,24)$ y por otra el mecanismo defensivo con que cuentan las células del carcinoma renal basado en la presencia de glicoproteina P (producto del gen MDR-1) que está implicada en los mecanismos de bombeo de productos tóxicos intracelulares al exterior de las células, y que en cierto modo explica la resistencia del carcinoma de células renales a la quimioterapia (25).

De conseguirse una elevadísima concentración de estos fármacos en el seno de un tumor renal, eludiendo los efectos sistémicos de los mismos, llegaría a hacerse ineficaz el mecanismo de bombeo defensivo de estas células y terminarían muriendo. Esta es la hipótesis que nos ha movido a investigar la forma de concentrar en el seno del tumor dosis masivas de un quimioterápico transportado por nanopartículas.

El hecho de que en nuestra investigación hayamos elegido como fármaco antitumoral a la Doxorrubicina obedece únicamente a que este producto había sido ya evaluado previamente en combinación con nanopartículas magnéticas $(26,27)$. El uso de la doxorrubicina por vía sistémica tiene al igual que el resto de los fármacos antitumorales el inconveniente su elevada toxicidad, que en su caso se manifiesta especialmente a nivel del músculo cardiaco.

En el futuro y sirviéndonos de ligandos específicos en la superficie de nuestras nanopartícula podremos vehiculizar con las misma inmunomoduladores u otros nuevos fármacos de diseño molecular que muestren una mayor efectividad en el tratamiento del carcinoma renal. 
Las nanopartículas de óxido de hierro son bien toleradas por el cuerpo y se degradan progresivamente con el tiempo (28). El tamaño de las partículas que suelen utilizarse más frecuentemente en aplicaciones biomédicas está por debajo de una micra, y usualmente son menores de 500 nanómetros. Las partículas más pequeñas suelen tener una gran área de superficie activa y baja fuerza de cohesión, algo que previene su agregación antes de aplicarles un campo magnético. Por el contrario, ellas tienen una baja susceptibilidad magnética e imanación de saturación, lo que hace que presenten una peor respuesta magnética. De aquí que se hace necesario ajustar los requerimientos para mantener un buen control magnético de las mismas en la entrega de la carga farmacológica que transportan.

Aunque nosotros hemos realizado los tests básicos recomendados para el estudio de biocompatibilidad de superficies, procurando adaptarlos para estudiar las nanopartículas circulantes en el torrente sanguíneo, hacemos constar que hasta el momento actual no se han definido bien los requisitos de los ensayos destinados a la valoración de ferrofluidos. En nuestra opinión, las nanopartículas circulantes deberían ser testadas mediante reología y estudios de activación plaquetaria, de coagulación y del posible daño endotelial.

A pesar de que los resultados iniciales con nuestro modelo quirúrgico experimental son satisfactorios, intentamos ahora aminorar aún más el trauma ocasionado por el implante de la diana magnética. Para ello estamos buscando un punzón de $9 \mathrm{Fr}$ que permita el implante de dos imanes cilíndricos de diámetro más reducido $(3 \mathrm{~mm}$ de diámetro $\times 1,5 \mathrm{~mm}$ de altura). El gradiente de campo magnético obtenido de esta forma equivaldría aproximadamente a la de la diana magnética que actualmente utilizamos.

Estamos diseñando también un procedimiento percutáneo, exclusivamente dirigido por radioscopia, para poner extraer las dianas magnéticas tras haber concluido la fase terapéutica.

El modelo experimental nos ha permitido comprobar que el implante laparoscópico de una diana magnética en el parénquima renal es un procedimiento fácilmente reproducible para cualquier urólogo que domine la laparoscopia. Que la tolerancia a corto y medio plazo de las dianas magnéticas en el seno del parénquima renal es buena. Su situación de la diana, una vez insertada, se controla fácilmente a lo largo del periodo postoperatorio mediante ecografía abdominal. Uno de los puntos más importantes de este estudio es la constatación histopatológica de la distribución y tolerancia de las nanopartículas , a cor- to y medio plazo, en ambos riñones, hígado, bazo, pulmón, ganglios y médula ósea.

En los cortes histopatológicos se observa un mayor cúmulo de nanopartículas en las proximidades de la diana magnética, pero sobre todo hemos observado que éstas llegan a contactar y adherirse firmemente a ésta resultando difícil separarlas dada su alta capacidad magnética.

Sin embargo, también hemos constatado presencia de nanopartículas repartidas en otros órganos, incluyendo al riñón contralateral. No hemos observado en los animales con supervivencias más largas que se produjera una mayor concentración de nanopartículas en la diana magnética, lo que nos hace pensar que la captación de las mismas se hace en los primeros momentos tras haber sido inyectadas intravenosamente.

\section{CONCLUSIONES}

La escasa respuesta de las células del carcinoma renal a la quimioterapia es ya conocida y está relacionada con su propia capacidad de autodepuración tóxica

La elevada toxicidad a nivel sistémico y la escasa respuesta obtenida hacen que la quimioterapia actual aporte muy pocos beneficios al tratamiento del hipernefroma, por lo que no se considera a ésta como un recurso de muy escasa utilidad.

Para conseguir contrarrestar el efecto de bombeo de substancias tóxicas al exterior que tienen las células del cáncer renal haría falta incrementar la dosis del fármaco hasta niveles que resultarían incompatibles con la vida.

La posibilidad de obtener localmente muy altas tasas de quimioterapia antitumoral y de poder ir renovando esta dosis dentro del propio tumor abre, desde el punto de vista teórico, nuevas esperanzas al tratamiento médico del hipernefroma.

Con el recurso de la nanotecnología se han abierto nuevos cauces para el transporte y entrega de fármacos. Las nanopartículas utilizadas con estos fines pueden focalizarse hacia el tumor mediante procedimientos físicos, químicos o inmunológicos.

El implante percutáneo intratumoral de dianas magnéticas permite focalizar hacia las mismas nanopartículas ferro carbonosas cargadas con un fármaco antitumoral.

El mayor obstáculo que se presenta ante esta nueva forma de administración de fármacos es la ba- 
rrera fisiológica del sistema retículo endotelial, que captura un alto porcentaje de las nanopartículas, impidiendo que lleguen al punto focalizado.

Nuestro equipo de investigación se centra actualmente en conseguir un blindaje temporal de las nanopartículas, que les permita eludir el ataque del sistema inmunocelular sin mermar el resto de sus funciones.

\section{BIBLIOGRAFÍA y LECTURAS RECOMENDADAS ( ${ }^{*}$ lectura de interés $y^{* *}$ lectura fundamental)}

1. WIDDER, K. J.; SENYEL, A. E.; SCARPELLI, G. D.: "Magnetic microspheres: a model system of site specific drug delivery in vivo". Proc Soc Exp Biol Med. 158:141,1978.

2. LUBBE, A. S.; BERGEMANN, C.; HUHNT, W. y cols.: "Preclinical experiences with magnetic drug targeting: tolerance and efficacy". Cancer Res. 56: 4694, 1996.

**3. ALEXIOU, C.; ARNOLD, W.; KLEIN, R. J. Y cols. "Locoregional cancer treatment with magnetic drug targeting". Cancer Research 60: 6641, 2000.

4. PANKHURST, Q. A.; CONNOLLY, J.; JONES, S. K. y cols. "Applications of magnetic nanoparticles in biomedicine”. Phys. D: Appl. Phys. 36 R167-R181 GUPTA, A. K. y GUPTA, M.: "Synthesis and surfa-

*5. ce engineering of iron oxide nanoparticles for biomedical applications". Biomaterials 26: 3995, 2005. KALAMBUR, V. S.; HAN, B.; HAMMER, B. E. y cols. "In vitro characterization of movement, heating

6. and visualization of magnetic nanoparticles for biomedical applications". Nanotechnology, 16:1221, 2005.

**7. SHINKAI, M.: "Functional magnetic particles for medical application". J. Biosci Bioeng 94: 606, 2002.

*8. LÜBBE, A. S.; ALEXIOU, C.; BERGEMANN, C.: "Clinical applications of magnetic drug targeting". J. Surgical Res. 95: 200, 2001.

**9. ALEXIOU, C.; JURGONS, R.; SCHMID, R. y cols.: "In vitro and in vivo investigations of targeted chemotherapy with magnetic nanoparticles". J. Mag Mag Mat. 293: 389, 2005.

10. AVILÉS, M. O.; EBNER, A. D.; CHEN, H. y cols. "Theoretical analysis of a transdermal ferromagnetic implant for retention of magnetic drug carrier particles”. J. Mag Mag Mat. 293: 605, 2005.

11. CHEN, H.; EBNER, A. D.; KAMINSKI, M. D. y cols. "Analysis of magnetic drug carrier particle capture by a magnetizable intravascular stent -2 : Parametric study with multi-wire two-dimensional model". J. Mag Mag Mat. 293: 616, 2005.

*12. ROSENGART, A. J.; KAMINSKI, M. D.; CHEN, H. y cols. "Magnetizable implants and functionalized magnetic carriers: A novel approach for nonin- vasive yet targeted drug delivery". J. Mag Mag Mat. 293: 633, 2005.

*13. YELLEN, B. B.; FORBES, Z. G.; HALVERSON, D. S. G. y cols. "Targeted drug delivery to magnetic implants for therapeutic applications". J. Mag Mag Mat. 293: 647, 2005.

14. VALDIVIA URÍA, J. G.: "Cirugía mínimamente invasiva". ENE Ediciones, Madrid ISBN: 84-8539547-6. 1993.

15. KRÄTSCHMER, L. D.; LAMB, K.; HUFFMAN, F. Y cols.: "Solid C60: a new form of carbon". Nature 347: 354, 1990.

*16. FERNÁNDEZ-PACHECO, R.; IBARRA, M. R.; VALDIVIA-URÍA, J. G. y cols. "Carbon coated magnetic nanoparticles for local drug delivery using magnetic implants". Nanotech 1:144, 2005.

17. CERRI, G.; DE GENNARO; BONFERONI, M. C. y cols. "Zeolites in biomedical application: Zn-exchanged clinoptilolite-rich rock as active carrier for antibiotics in anti-acne topical therapy". Applied Clay Science 27: 141, 2004.

18. KUZNETSOV, A. A.; FILIPPOV, V. I.; KUZNETSOV, O. A. Y cols. "New ferro-carbon adsorbents for magnetically guided transport of anti-cancer drugs". J. Mag Mag Mat 194: 22, 1999.

19. NAGESWARI, K.; BANERJEE, R.; GUPTE R, V. y cols. "Hemorheological parameters for biocompatibility evaluation". J. Biomater Appl. 13: 74, 1998.

20. REINHART, W. H.; PLEISCH, B.; HARRIS, L. G. y cols. "Influence of contrast media (iopromide, ioxaglate, gadolinium-DOTA) on blood viscosity, erythrocyte morphology and platelet function". Clin. Hemorheol Microcirc. 32: 227, 2005.

21. YAGODA, A.: "Chemotherapy of renal cell carcinoma: 1983 - 1989”. Semil. Urol. 7: 199, 1989.

22. YAGODA, A.; ABI-RACHED, B.; PETRYLAK, D.: "Chemotherapy for avanced renal cell carcinoma". Sem. Oncology 22: 42, 1995.

23. HRUSESKY, W. J. M.; VON ROEMELING, R.; LANNING, R. M. y cols. "Circadian -shaped infusions of floxuridine for progressive metastatic renal carcinoma”. J. Clin. Oncol. 8: 1504, 1990.

24. DEXEUS, F. H.; LOGOTHETIS, C. J.; SELLA, A. y cols. "A circadian infusión of floxuridine in patients with metastatic renal cell carcinoma". J. Urol. 146: 709, 1999.

*25. MICKISCHG, H. y WORD, J. "Chemorresistance of renal cell carcinoma: 1986 - 1994". World J. Urol. 12: 214, 1994.

26. WU YI, S.; GONG CHENG, X.; ZA ZHI, X. “A study on preparating of magnetic doxorrubicin nanoparticles and its heat effect under a magnetic field". Medscape (ISSN 1001-5515) 20:463, 2003.

*27. JAIN, T. K.; MORALES, M. A.; SAHOO, S. K. y cols. "Iron oxide nanoparticles for sustained delivery of anticancer agents". Molec. Pharm. 2: 194, 2005.

28. OCÓN, E.; POULIQUEN, D.; OCÓN, P. y cols. "Biodegradation of magnetite dextran nanoparticles in the rat. A histologic and biophisical study". Lab. Invest. 71: 895, 1994. 